\title{
Nanoscale Probing of Bandgap States on Oxide Particles Using Electron Energy-Loss Spectroscopy
}

Qianlang Liu ${ }^{1}$, Katia March ${ }^{2}$ and Peter A. Crozier*1

1. School for the Engineering of Matter, Transport and Energy, Arizona State University, 85287 Arizona, USA.

2. Laboratoire de Physique des Solides, Bâtiment 510, Université Paris-Sud, 91405 Orsay Cedex, France.

* Corresponding author e-mail: CROZIER@asu.edu 


\begin{abstract}
:
Surface and near-surface electronic states were probed with nanometer spatial resolution in $\mathrm{MgO}$ and $\mathrm{TiO}_{2}$ anatase nanoparticles using ultra-high energy resolution electron energy-loss spectroscopy (EELS) coupled to a scanning transmission electron microscope (STEM). This combination allows the surface electronic structure determined with spectroscopy to be correlated with nanoparticle size, morphology, facet etc. By acquiring the spectra in aloof beam mode, radiation damage to the surface can be significantly reduced while maintaining the nanometer spatial resolution. $\mathrm{MgO}$ and $\mathrm{TiO}_{2}$ showed very different bandgap features associated with extrinsic species on the surface/sub-surface layer of the nanoparticles. Spectral simulations based on dielectric theory and density of states models showed that a plateau feature found in the pre-bandgap region in the spectra from (100) surfaces of $60 \mathrm{~nm} \mathrm{MgO}$ nanocubes is consistent with a thin hydroxide surface layer. The spectroscopy shows that this hydroxide species gives rise to a broad filled surface state at $1.1 \mathrm{eV}$ above the $\mathrm{MgO}$ valence band. At the surfaces of $\mathrm{TiO}_{2}$ nanoparticles, pronounced peaks were observed in the bandgap region, some of which were associated with surface defects from impurities and oxygen vacancies. The spectral interpretation suggests that half-filled surface states at about 1.6 and $2.4 \mathrm{eV}$ above the valence band were present.
\end{abstract}

\title{
Keywords:
}

Bandgap states, surface states, dielectric property, electronic structure, nanoparticle 


\section{Introduction}

Ever since the existence of specific electron states associated with crystal surfaces was first illustrated by Tamm [1], various researchers have conducted experimental characterization and theoretical calculations of surface states, especially in the fields of solid state physics and catalysis [2-6]. By one definition, a surface state is an electronic state in which the probability amplitude of an electron decreases exponentially with increasing distance from the surface (outside or inside) [7]. The origin of surface states can be categorized into two types: intrinsic, meaning states of a free surface without any foreign atom, and extrinsic, including the ones associated with absorbed atoms and/or surface defects [8,9]. In more general definitions, electronic states can also be associated with surface and subsurface species which may form layers that are distinct from the underlying substrate phase. The focus of this paper will be on these latter structures concentrating on interpreting the extrinsic states detected on oxide nanoparticle surfaces, particularly $\mathrm{MgO}$ and $\mathrm{TiO}_{2}$, probed using a focused electron beam with nanometer resolution in an electron microscope.

The character of surface states influences nanoparticle interactions with other particles or adsorbate species. For example, in heterogeneous catalysis, nanoparticulate systems are usually preferred since the absorption and desorption of the reactants and products, as well as the breaking and forming of chemical bonds can occur more efficiently on large surface areas. The electronic structure on the surfaces/interfaces determines the charge transfer and controls the selectivity and kinetics of the chemical reactions. Heterogeneity and defects very often will occur during the preparation and processing of particulate based catalysts giving rise to different types of extrinsic surface states. Characterizing the local nanoscale variation in oxide surface states is challenging but important for developing a fundamental understanding of functionality. 
Experimentally, many techniques have been developed to measure electronic structures of materials' surfaces including ultraviolet photoemission spectroscopy (UPS) [10], reflection electron energy loss spectroscopy (REELS) [11-13], metastable impact electron spectroscopy (MIES) [10,14], scanning tunneling microscopy (STM) [15] and surface photovoltage spectroscopy (SPS) [16]. These techniques probe different aspects of the materials' electronic structure, usually requiring flat sample surfaces for better data quality, but, except for STM, do not have the ability of imaging sample microstructure simultaneously. Here we employ a novel form of monochromated electron energy-loss EELS [17], giving an energy resolution of $15 \mathrm{meV}$ or better as well as a substantially smaller zero loss peak tail compared to regular cold-FEG or Schottky FEG instruments, to detect and characterize electronic states on or near the surfaces of oxide nanoparticles. The energy-loss capability is coupled to an aberration-corrected STEM, allowing the ultra-high energy resolution spectroscopy to be performed with a sub-nanometer electron probe. With this configuration, atomic level electron imaging can provide high spatial resolution information on surface morphology while EELS can provide the local electronic properties of the near-surface region.

Most energy-loss spectra are recorded by transmitting a fast electron beam through the sample (Figure 1a). Recording energy-loss spectra with a sub-nanometer electron probe can permit electronic structure to be probed with atomic resolution $[18,19]$. However, the high incident electron energy and the relatively large electron dose associated with this high spatial resolution analysis may damage the structure under observation. Radiation damage effects are particularly pronounced on surfaces because the open structure makes mass loss more facile [20]. When the electron beam passes through the sample, two types of radiation damage mechanisms are possible: knock-on (direct displacement of the nucleus by the fast electron) and radiolysis 
(ionization by the fast electron), which are described in detail elsewhere [21,22]. An alternative approach to spectral acquisition is the so-called "aloof beam" mode where the electron probe is parked some distance away from the surface of the sample [23-25] (Figure 1b). The delocalized component of the electron-solid interaction allows the electronic states at or near the surface to be excited even when the probe is positioned outside the sample. In aloof mode, the knock-on damage process is essentially turned off and radiolytic damage can be greatly reduced depending on the distance between the specimen surface and the electron beam. This opens up the possibility for nanometer resolution detection of surface states because radiation damage is significantly suppressed and the EELS signal is strong only when the probe is within a few nanometers of the surface.

In this work, we first investigated the extrinsic surface states associated with the (100) facets of $\mathrm{MgO}$ nanocubes. Surface species are likely to give rise to states within the bandgap and the well-defined morphology and wide bandgap make $\mathrm{MgO}$ an ideal initial test system for the aloof beam EELS approach. Using low-energy REELS, Henrich et al have observed an intrinsic surface transition signal at $6.2 \mathrm{eV}$ and a $2.3 \mathrm{eV}$ peak possibly associated with surface defects in $\mathrm{MgO}$ [12]. Moreover, both experimental and theoretical work indicates that $\mathrm{MgO}$ readily absorbs water in ambient atmosphere and water molecule dissociation and hydroxyl species formation often occur on surface steps [26]. This hydroxylation process generates a source of extrinsic surface states on $\mathrm{MgO}$ nanocubes. The relative simple $\mathrm{MgO}$ system is a direct bandgap insulator and is ideal for exploring the advantages and disadvantages of both a dielectric function and a density of states (DOS) approach to spectral interpretation.

The EELS approach was then applied to probe the more complex surface electronic structures of $\mathrm{TiO}_{2}$ based photocatalyst nanoparticle system. Surface states on reducible oxides 
are believed to play a major role in the functionality of photocatalysts but it is currently very difficult to characterize on real high surface area systems composed of nanoparticles. In this experiment, we investigate the character of states on a supported metal catalyst consisting of $\mathrm{Ni}$ metal nanoparticles loaded onto anatase. $\mathrm{TiO}_{2}$ functionalized with $\mathrm{Ni}$ based co-catalysts show interesting behaviors in photocatalytic/photocorrosion reactions [27,28]. In photocatalytic processes, electron-hole pairs, generated by photon absorption in $\mathrm{TiO}_{2}$, migrate to the surface and may participate in catalytic reactions. Surface states play a vital role in photocatalytic reactions because they can serve as charge traps to increase the lifetime of a carrier and prevent recombination of electrons and holes [29]. Such traps may also lower the overpotential of the photoelectron and negatively impact catalytic properties.

\section{Material and Methods}

Materials: $\mathrm{MgO}$ is obtained by collecting the product particles while combusting $\mathrm{Mg}$ ribbon in air and then exposed to water vapor. A portion of the $\mathrm{MgO}$ particles were then dispersed in DI water and heated to $70{ }^{\circ} \mathrm{C}$ for about $10 \mathrm{~h}$ to produce $\mathrm{Mg}(\mathrm{OH})_{2}$. Commercial $\mathrm{TiO}_{2}$ anatase particles $(99.8 \%)$ were purchased from Sigma-Aldrich. Ni metal was loaded on $\mathrm{TiO}_{2}$ substrates using wetness impregnation followed by high temperature reduction at $450{ }^{\circ} \mathrm{C}$ in flowing $\mathrm{H}_{2}$.

An aberration-corrected NION UltraSTEM 100 microscope coupled with a monochromator and a Gatan Enfinium spectrometer was employed to acquire all the spectra. The microscope was operated at $60 \mathrm{kV}$ with an energy dispersion of $5 \mathrm{meV}$ per channel. Full width half maximum (FWHM) of the zero-loss peak was better than $25 \mathrm{meV}$. Convergence angle $\alpha$ was 
$30 \mathrm{mrad}$, and detector collection angle $\beta$ was $15 \mathrm{mrad}$ with $1 \mathrm{~mm}$ EELS entrance aperture. Gatan Digital Micrograph software was used to process the data. To facilitate interpretation of the energy-loss spectra, X-ray photoelectron spectroscopy (XPS) was performed with a VG 220i-XL to detect the average elemental composition on the surfaces of commercial anatase powder.

For the $\mathrm{MgO}$ system, spot spectra were acquired where the beam was positioned either in bulk $\mathrm{MgO}$ or $4 \mathrm{~nm}$ away from the surface and the acquisition time was $50 \mathrm{~s}$ to maximize the signal to noise ratio. On the other hand, EELS line scans were performed for the $\mathrm{TiO}_{2}$ system, where the beam was scanned from the vacuum (about $30 \mathrm{~nm}$ outside the sample) into bulk $\mathrm{TiO}_{2}$. At each scan point, the acquisition time was typically $30-40$ s. Point spectra in one line scan where the electron beam was outside the sample were integrated to improve the signal-to-noise ratio in the bandgap region, and labelled as the extracted aloof spectrum. Point spectra in the same line scan where the electron beam was in the bulk $\mathrm{TiO}_{2}$ were also integrated and labelled as the extracted transmission spectrum.

\section{Results and Discussion}

In the most common form of EELS acquisition, the electron beam passes through the TEM specimen (Figure 1a) whereas in the aloof beam configuration, the beam is positioned some distance outside the surface of the sample as shown in Figure 1b. The distance of the beam from the surface is typically called the impact parameter $b$. Although the electron beam does not enter the sample in this case, it can still excite the electrons in the material via the Coulomb field interaction. In STEM, the electron beam is converged to a small spot using convergent illumination. Considering the specimen thickness $t$ and an electron beam with a convergence 
angle $\alpha$, the aloof beam geometry will apply only when the impact parameter $b \geq \frac{t}{2} \times \tan (\alpha)$. This condition must be satisfied in the STEM to ensure that no electrons enter any part of the sample.

For the experimental data presented in this paper, a $30 \mathrm{mrad}$ convergence angle was used and sample thicknesses were usually smaller than $100 \mathrm{~nm}$ so, in general, the aloof condition applied when the beam was at least $1.5 \mathrm{~nm}$ away from the surface. A $4 \mathrm{~nm}$ impact parameter was often used when aloof beam EELS was acquired from $\mathrm{MgO}$. For $\mathrm{TiO}_{2}$, EELS line scans were often performed during which the beam started some distance outside the sample and spectra were acquired at each point as the beam was stepped towards and into the sample. Such data series contain aloof and transmission EELS. The signal in the bandgap is very weak so to improve signal-to-noise, spectra from a line scan series were often summed either over the vacuum points or the transmission points.

\subsection{States on (100) MgO Nanoparticles Surface}

Transmission and aloof spectra from an $\mathrm{MgO}$ nanoparticle are shown in Figure 2. A highangle annular dark field image (HAADF) shows the nanocubes terminated with (100) surfaces which are well known to be the low energy surface facet [30]. The beam was positioned on top of a nanocube for the transmission spectrum, and $4 \mathrm{~nm}$ away from the nanocube for the aloof spectrum. Both raw spectra (Figure 2a) show an abrupt increase in intensity at $7.3 \mathrm{eV}$ corresponding to the conduction band edge and a bandgap of $7.3 \mathrm{eV}$ is in agreement with literature value [31]. The tail on the zero-loss peak was best fitted by a first order lognormalpolynomial background model available in the Gatan Digital Micrograph software, and the background subtracted spectra are compared in Figure 2b. For a $60 \mathrm{kV}$ electron, significant 
Cherenkov will only occur when the refractive index of the material is larger than 2.24 [32] and the refractive index of $\mathrm{MgO}$ is 1.7. Thus in this case we do not expect significant energy-losses due to Cherenkov radiation. Interestingly, the aloof spectrum shows an additional continuous intensity rise starting from $5.6 \mathrm{eV}$ leading to a plateau which merges into the conduction band onset at $7.3 \mathrm{eV}$. There is some evidence for a similar effect in the transmission spectrum but of much smaller relative intensity. The high intensity of this plateau in the aloof beam spectrum suggests that this spectral feature is associated with surface state transitions. The weaker feature observe in the transmission spectrum is associated with the beam passing through the top and bottom surfaces of the cube.

To investigate the origin of this spectral feature we employ dielectric theory to simulate the energy-loss spectra for transmission and aloof beam geometries. The simulations employed $\mathrm{MgO}$ dielectric properties derived from reflectance measurements [31] and slightly adjusted to fit with the experimental bandgap onset. Dielectric based theories have been developed to simulate transmission and aloof beam spectra [24,25,33]. Since we are using $60 \mathrm{kV}$ electrons for the current experiments we can ignore relativistic effects. In the non-relativistic limit, treating the experimental data as single scattering distribution, the transmission spectra, $\mathrm{S}(\mathrm{E})$, can be simulated using the energy loss function $\operatorname{Im}(-1 / \varepsilon)$, where $\varepsilon$ is the energy dependent complex dielectric function [32,34]. For the bandgap region and sample thickness of interest here, plural scattering effects are small and are neglected.

For a bulk terminated sample illustrated in Figure 3a, the aloof spectrum for an electron travelling parallel to surface at a distance of $b$ (impact parameter) can be written as [24]

$$
\mathrm{I}(\mathrm{E}) \propto \operatorname{Im}\left(-\frac{1}{\varepsilon+1}\right) \times K_{o}\left(\frac{2 \omega b}{v}\right)
$$


where $\mathrm{I}(\mathrm{E})$ is the intensity in the EELS spectrum, $K_{o}$ is the zero-order modified Bessel function, $\omega$ is the frequency corresponding to each energy loss, $v$ is the velocity of the fast electron in the beam. One limitation of the model is that it assumes the beam is parallel to the sample surface while in reality the STEM probe is a convergent beam. However, it has been demonstrated this approximation is reasonable and does not significantly affect the shape of the EELS spectra [35].

The simulated transmission and aloof spectra of bulk $\mathrm{MgO}$ were calculated and compared with experimental transmission data in Figure 4. Each spectrum was normalized so that the signal intensity at $7.9 \mathrm{eV}$ was set to unity. The dielectric approach produces a good match in the overall shape between the simulated spectra and the experimental data reproducing the two peaks at about 7.9 and $11.8 \mathrm{eV}$. No pre-bandgap intensity was seen in the simulated aloof spectrum from pure $\mathrm{MgO}$ suggesting the additional intensity at $5.6 \mathrm{eV}$ is associated with extrinsic states caused by the presence of a surface phase.

The most likely origin of the surface phase is the presence of hydroxyl species leading to a possible thin layer of magnesium hydrate. To simulate the influence of a surface phase, a more sophisticated dielectric model is required which explicitly includes a thin film on a bulk substrate. Such a model is illustrated in Figure $3 \mathrm{~b}$ in which the surface phase is represented by a thin layer of thickness $2 \mathrm{a}$ with dielectric function $\varepsilon_{1}$ on a substrate material with dielectric function $\varepsilon$. In the non-relativistic limit, the aloof beam spectrum, I(E), for an electron travelling parallel to the surface with impact parameter b is given by [24]

$$
\mathrm{I}(\mathrm{E}) \propto\left\{\mathrm{F}\left(\varepsilon, \varepsilon_{1}, \mathrm{~K}, \mathrm{a}\right) \times \exp (-2 \mathrm{~K} \times \mathrm{b})\right\}
$$




$$
\begin{gathered}
K^{2}=q^{2}+\left(\frac{\omega}{v}\right)^{2} \\
\mathrm{~F}\left(\varepsilon, \varepsilon_{1}, \mathrm{~K}, \mathrm{a}\right)=\operatorname{Im}\left\{\left[\left(\varepsilon_{1}+\varepsilon\right)\left(\varepsilon_{1}-1\right) \exp (2 K a)-\left(\varepsilon_{1}-\varepsilon\right)\left(\varepsilon_{1}+1\right) \exp (-2 K a)\right]\right. \\
\left.\times\left[\left(\varepsilon_{1}+\varepsilon\right)\left(\varepsilon_{1}+1\right) \exp (2 K a)-\left(\varepsilon_{1}-\varepsilon\right)\left(\varepsilon_{1}-1\right) \exp (-2 K a)\right]^{-1}\right\}
\end{gathered}
$$

where $\mathrm{q}$ is the momentum transfer parallel to the plane of the interface. The geometry of this socalled infinite slab model is an approximation because in reality, the top and bottom of the substrate should also be coated with $\varepsilon_{1}$ surface dielectric layers. But their contribution to the aloof EELS spectrum is small as they are further away from the beam.

To explore the hydroxide hypothesis, it is necessary to know the dielectric function for $\mathrm{Mg}(\mathrm{OH})_{2}$. No accurate experimental dielectric data of $\mathrm{Mg}(\mathrm{OH})_{2}$ was found from the literature so an estimate of the dielectric function was made from experimental energy-loss spectra from a $\mathrm{Mg}(\mathrm{OH})_{2}$ reference compound. The $\mathrm{Mg}(\mathrm{OH})_{2}$ had a non-uniform morphology and is extremely beam sensitive. This made it impossible to apply standard Kramer-Kronig methods to determine the dielectric function [36]. Experimental EELS spectra of $\operatorname{Mg}(\mathrm{OH})_{2}$ were acquired in aloof mode to minimize the radiation damage. We assumed that the imaginary part of the dielectric function was proportional to the spectrum and real part was adjusted so that spectrum simulated from eqn (1) matches the experimental curve as shown in Figure 5a. The values of the dielectric data were set so that the refractive index matched the value that has been reported [37]. To perform the simulation, the impact parameter $\mathrm{b}$ was fixed at $4 \mathrm{~nm}$, while the layer thickness $2 \mathrm{a}$ was changed to match the experiment. A thickness of less than $2 \mathrm{~nm} \mathrm{Mg}(\mathrm{OH})_{2}$ gave the best fit to the experiment data shown in Figure 5b.

The dielectric function derived from bulk $\mathrm{Mg}(\mathrm{OH})_{2}$ sample had a shape that matched the experimental spectrum but there is uncertainty in the absolute value introducing considerable 
uncertainty in the thickness of the surface layer. Moreover, while the dielectric approach provides information on excitation energies of the surface it does not directly provide information on the energy of the surface states with respect to the $\mathrm{MgO}$ band edges. An alternative approach is to simulate the energy-loss spectra using an approximation that starts from knowledge of the DOS. With this approach, the spectral intensity within the bandgap region arises from specific states that are present within the bandgap. The energy, width and number of the states are adjusted to match the experimental observations.

The DOS approach works in part because for an insulator, the aloof beam spectrum can be approximately decomposed into bulk and surface contributions. For the insulators of interest here, examination of the real and imaginary parts of the dielectric function shows that $\operatorname{Im}\left(-\frac{1}{\varepsilon+1}\right) \sim \operatorname{Im}\left(\frac{-1}{\varepsilon}\right)$. Thus the bulk contribution to the aloof beam spectrum closely resembles the transmission spectrum with the signal arising from the higher energy-loss attenuated because they are more strongly localized. The localization attenuation is contained in the Bessel function term of eqn (1). Thus for the oxides of interest here, the energy-loss intensity can be approximately re-written in the form

$$
\mathrm{I}(\mathrm{E}) \propto \operatorname{Im}\left(-\frac{1}{\varepsilon}\right) \times K_{o}\left(\frac{2 \omega b}{v}\right)
$$

Figure 4 shows that a simulated spectrum from eqn (3) closely matches the spectrum determined from the more rigorous eqn (1). Thus the bulk contribution to the aloof beam spectrum strongly resembles the transmission spectrum.

The transmission single scattering distribution, $\mathrm{S}(\mathrm{E})$ can also be interpreted in terms of a convolution between the valence and conduction band DOS through the equation[32] 


$$
\mathrm{S}(\mathrm{E}) \propto \rho_{V B}(E) * \rho_{C B}(E)
$$

where the right hand side, the so-called joint density of states (JDOS), is the convolution of the initial (occupied) projected density of states (PDOS) in the valence band, $\rho_{V B}(E)$, with the final (empty) PDOS in the conduction band, $\rho_{C B}(E)$. The convolution is usually performed following the dipole selections rules which dominate the features in the energy-loss spectrum. The JDOS approach does not take many body effects (like excitons) into account but can often provide a guide to identify the origin of electronic structure features in the spectrum. This simple approach has been successfully employed to locate the position and width of bandgap states with respect to the conduction band edge in doped ceria [38]. The similarity between the aloof and transmission spectra for $\mathrm{MgO}$ suggests that this approach may be useful for the interpretation of surface states. In this interpretation, the bulk contribution to the aloof beam spectrum is assumed to arise from the bulk DOS and the surface contribution comes from states located within the bandgap. Spectral simulations using eqn(3) on oxides systems where surface layer thickness is varied justify this approach.

The DOS for MgO has been calculated by various researchers [39-41] and a firstprinciples calculation by $\mathrm{Xu}$ et al [42] was employed here, and slightly modified to better fit the experimental data (Figure 6a). Figure $6 \mathrm{~b}$ shows the derived JDOS obtained by convolving the PDOS following the dipole selection rules which approximately apply to EELS. Because the valence band is mainly $\mathrm{O} 2 \mathrm{p}$ and the conduction band is mainly $\mathrm{Mg} 3 \mathrm{~s}$, the $\mathrm{O} 2 \mathrm{p} \rightarrow \mathrm{Mg} 3 \mathrm{~s}$ transition predominates and determines the shape of the JDOS. Compared to the experimental data, the peak at $7.9 \mathrm{eV}$ in the experiment is missing. Sobolev and others have indicated that this peak is associated with exciton states in $\mathrm{MgO}$ which could only be observed when the material is in the excited state $[31,42,43]$. This exciton phenomenon is not included in the one electron 
ground state calculation of the DOS data and will introduce some uncertainty in the number of surface states present.

The bulk contribution to the aloof beam spectra can be simulated by using eq(4). The surface contribution will then arise by inserting additional states into the bandgap region. It is informative to consider the effect of introducing a bandgap state on the form of the energy-loss spectrum. A fully occupied bandgap state will give transitions into the empty conduction band and the convolution shows that this will yield a spectral intensity distribution in the bandgap region that looks like the conduction band DOS. A fully unoccupied state will be associated with transitions from the valence band so it will give rise to an intensity distribution in the bandgap that resembles the valence band DOS. Thus we see that a sharp bandgap state will essentially give a spectrum that looks either like the conduction or valence band DOS in the bandgap.

The theoretical data in figure 6 shows that the DOS for both the valence and conduction band varies rapidly within the $2-3 \mathrm{eV}$ of the band edges. However, the experimental data of Figure $2 \mathrm{~b}$ show a more slowly varying smooth increase suggesting that the bandgap states must be broad. The simulated spectrum with a broad filled bandgap state is shown in Figure 7. As expected the exciton feature at $7.9 \mathrm{eV}$ was not reproduced but, for the intensity below the bandgap, the best agreement with the experiment was obtained with a Gaussian shaped filled state centered at $1.1 \mathrm{eV}$ above the valence band with a FWHM of $0.7 \mathrm{eV}$. It is reasonable that the surface states associated with the hydroxide layer are broad. Narrow energy states are associated with isolated point defects such as oxygen vacancies or impurities/dopants and as the defect densities increases, these state become broader. Finding that a relatively broad defect state fits the experimental data qualitatively agrees with the dielectric interpretation of a continuous layer of $\mathrm{Mg}(\mathrm{OH})_{2}$ on the surface. The advantage of the DOS approach compared to the dielectric 
interpretation is that it provides an estimate of the energy and width of the defect state with respect to bulk band edges.

\subsection{States on Anatase Nanoparticle Surfaces}

The $\mathrm{Ni} / \mathrm{TiO}_{2}$ system exhibits a variety of less well-defined surface facets with different populations of Ni metal particles. This is more complicated to characterize but also is more typical of nanoparticle systems of relevance to fields such as catalysis and corrosion. Figure 8 shows a HAADF image of a typical area showing $10-20 \mathrm{~nm}$ Ni particles loaded on anatase particles $150 \mathrm{~nm}$ in size. While surface states associated with the Ni metal are of interest, the focus of the current work is on the oxide support. The energy-loss line scans discussed here are recorded at least $20 \mathrm{~nm}$ from the metal particles and are characteristic of the oxide surfaces.

Figure 8 shows summed selected transmission and aloof spectra extracted from the line scan going from the vacuum into anatase. The transmission spectrum from bulk $\mathrm{TiO}_{2}$ shows a bandgap onset at about $3.4 \mathrm{eV}$ with a relatively featureless bandgap region. In this case, the decreasing intensity is associated with tails on the zero-loss peak and possible Cherenkov losses (refractive index $\sim 2.43$ [44]). The bandgap region from the aloof beam spectra is very different showing a series of peaks labelled $1-5$ with corresponding energies of $0.85,2.1,2.4,2.8$ and 3.2 $\mathrm{eV}$. The features are not present in the transmission spectrum and decay with impact parameter indicating that they are associated with the surface region of the nanoparticle.

The aloof beam spectra senses the local dielectric response of the surface layer of the $\mathrm{TiO}_{2}$ nanoparticles. For insulators, this response is approximately a combination of the response from the surface and bulk (e.g. see eqn (2) and (3)). Below the bulk bandgap, the spectrum from the substrate is zero and the aloof beam technique essentially measures the dielectric response of 
the surface layer. This region of the spectrum will contain surface excitonic effects and will show the energies of possible light absorption at the surface. In this case, the energy-loss spectrum shows that the surface of this $\mathrm{TiO}_{2}$ nanoparticle may absorb light in the visible region with two strong absorption peaks at 2.1 and $2.4 \mathrm{eV}$. This is consistent with the well-known coloration of initially white titania that occurs after reduction treatments $[45,46]$. This may allow us to map and characterize defect induced zones of visible light absorption on the surfaces of oxide nanoparticles.

We can also use a DOS approach to approximately simulate the bulk contribution from anatase (Figure 9a) [47]. The simulated transmission spectrum is shown in Figure $9 \mathrm{~b}$ and gives a reasonable fit with the experimental transmission spectrum. The presence of peaks in the bandgap region of Figure 8 indicate the presence of one or more narrow energy states within the bandgap. The number of peaks observed in the spectrum depends in part on the number of peaks in the relevant DOS. For example, for a narrow empty state within the bandgap, the shape of the spectral intensity distribution within the bandgap is a map of the valence band DOS.

The states within the bandgap possibly originated from the following two factors. First, the impurity elements in the commercial anatase could serve as a source of extrinsic bandgap states. In our case, a few atomic percent of $\mathrm{K}$ and $\mathrm{P}$ were detected on the surface of the anatase particles using XPS. It has been shown that $\mathrm{K}$ and $\mathrm{P}$ can associate with $\mathrm{TiO}_{2}$ surfaces as adsorbates or precipitates[48,49]. Also, the sample underwent a fairly aggressive reduction in $\mathrm{H}_{2}$ at $450{ }^{\circ} \mathrm{C}$ for 2 hours to create the Ni metal particles, which may result in impurities segregating to the surface layer. These are consistent with the fact that the bandgap features only show in aloof mode but not in transmission mode. Hardman et al. showed that adsorption of $\mathrm{K}$ on $\mathrm{TiO}_{2}$ (100) surface results in $\mathrm{Ti}^{3+}$ formation and charge transfer from $\mathrm{K} 4 \mathrm{~s}$ to $\mathrm{Ti} 3 \mathrm{~d}$ induces bandgap 
states [49]. A second possibility is that, during the heat treatment, the Ni metal particles will rapidly dissociate $\mathrm{H}_{2}$ and the resulting protons will then reduce the $\mathrm{TiO}_{2}$. At this temperature, the $\mathrm{TiO}_{2}$ has significant oxygen ionic conductivity and some vacancies will diffuse to subsurface sites [50]. On cooling, the top layer may partially re-oxidize but kinetic diffusion limitations will maintain significant oxygen vacancy concentration at subsurface sites. This suggests that there will be a high concentration of oxygen vacancies near the nanoparticle surface. Oxygen vacancies in $\mathrm{TiO}_{2}$ have been studied both experimentally and theoretically $[45,46,51,52]$. In one picture, the two electrons left behind when the oxygen is removed transfer to adjacent titanium atoms forming $\mathrm{Ti}^{+3}$ species. These electrons are effectively injected into an empty $\mathrm{Ti} 3 \mathrm{~d}$ state forming a partially filled band located $0.4-2 \mathrm{eV}$ below the conduction band $[45,46]$. $\mathrm{The}^{3+}$ species have been assigned to a $0.8 \mathrm{eV}$ feature in energy loss spectrum coupled to an STM [53]. It has also been calculated that an oxygen vacancy on the rutile (110) surface can introduce two localized $\mathrm{Ti}^{3+} 3 \mathrm{~d}^{1}$ states $1 \mathrm{eV}$ below the conduction band edge [52]. Thus both surface impurity elements and oxygen vacancies may result in $\mathrm{Ti}^{3+}$ species formation altering the surface electronic structure.

To explore these hypotheses, we simulated the energy-loss spectra using a partially filled state positioned below the conduction band. In this case, because of the dipole selection rules, we assumed that transitions from the defect state (d-like) to the conduction band (d-like) were weak and simulated only transitions from the valence band (p-like) into the defect state. The total spectrum is the sum of contributions from this defect state and a contribution from the bulk bandto-band transitions for anatase. There are variations in density functional theory (DFT) DOS calculations for anatase electronic structure [47,54,55]. It is not clear how the presence of impurities and oxygen vacancies in the subsurface region may perturb the DOS. In an initial 
simulation, we considered a single defect state $1.6 \mathrm{eV}$ above the top of the valence band with a FWHM of $0.19 \mathrm{eV}$ and used the DOS given in Figure 9a. The fit to the experimental data gave a good agreement to peaks 2 and 3 and a reasonable fit to peaks 4 and 5 in Figure 8. Peak 1 at $0.85 \mathrm{eV}$ was not reproduced in the initial simulation.

To simulate a spectrum that included all of the peaks it was necessary to introduce a second partially filled state within the bandgap at an energy of $0.85 \mathrm{eV}$ shifted from the first bandgap state. The peak at $0.85 \mathrm{eV}$ loss could then arise as a result of transitions between these two states. The spectrum simulated from this combination of states is shown in Figure 10. The best fit was obtained with two states positioned at 1.6 and $2.4 \mathrm{eV}$ above the valence band top with FWHM of $0.19 \mathrm{eV}$. The two states may still be d-states associated with different configuration of point defects. For the STEM collection and convergence angles employed here, non-dipole transitions will contribute weakly to the spectrum and d-d transitions have been observed previously in $\mathrm{NiO}$ [56]. In the present case, the sharpness of the two bandgap states allows this weaker transition probability to give a detectable peak in the spectrum at 0.85 . The intensity of states associated with the state at $1.6 \mathrm{eV}$ is about 5 times stronger than that at 2.4 suggests that the two different defect motifs are present in approximately a 5:1 ratio in the surface region.

The analysis of Figure 8 shows two defect states present near the surface region of the nanoparticle but other defect configurations may be present given the heterogeneous and rather disordered nature of the particle surfaces. Impurities and oxygen vacancies may be present at a range of concentrations and defects may cluster leading to different configurations of $\mathrm{Ti}^{3+}$ and $\mathrm{Ti}^{2+}$ species. The large number of possible defect configurations makes it unlikely that spectra from different particles will be identical. It would be interesting to investigate the variation in 
surface states with different reduction treatments using in situ EELS measurements. Moreover, in some spectra, not all peaks in the bandgap region can be simulated using the DOS approach. As shown in Fig. 11, similarly to the previous case, a series of sharp features within the bandgap were only present when the electron beam was outside or on the surface of the anatase particle. The peaks labelled 1-5 are at energies approximately similar to those observed in Figure 8 and can be fitted by inserting bandgap states at 1.8 and $2.5 \mathrm{eV}$. Two additional peaks (6 and 7) are also present which cannot be fitted with the DOS approach. It is possible that peaks 6 and 7 are not generated from defects in the material but are due to some other electron scattering phenomena such as Cherenkov or transition radiation. For example, Cowley has discussed transition radiation associated with electron channeling along surfaces of $\mathrm{MgO}$ cubes [57]. If such effects give rise to peaks in the bandgap region they will likely have a strong dependence on refractive index and crystal orientation. At present these additional peaks are not fully understood and are currently under investigation.

\section{Conclusions}

We have used ultra-high energy resolution EELS in a STEM to investigate the electronic states associated with the surfaces of oxide nanoparticles. By employing the so-called aloof beam mode, radiation damage to oxide surfaces can be suppressed while still allowing nanometer spatial resolution to be maintained. The surface states show up as features within the bandgap region of the spectrum which can be interpreted within either a dielectric function or DOS framework. The dielectric theory was applied to interpret the data from (100) surfaces of $60 \mathrm{~nm}$ $\mathrm{MgO}$ cubes and showed that the experimental spectrum was consistent with the presence of a hydroxide surface layer. The DOS approach showed that the overlayer was associated with a 
broad occupied state located $1.1 \mathrm{eV}$ above valence band of $\mathrm{MgO}$ substrate. The surfaces of $\mathrm{TiO}_{2}$ anatase nanoparticles showed a series of sharp peaks in the bandgap region suggesting the presence of narrow defect states within the bandgap. In this case two different states at approximate energies of 1.6 and $2.4 \mathrm{eV}$ above the valence band top were observed which were presumably associated with half-filled $\mathrm{Ti}^{3+} 3 \mathrm{~d}$ orbitals.

\section{Acknowledgements}

We would like to acknowledge Dr. Toshihiro Aoki (LeRoy Eyring Center for Solid State Science, Arizona State University) for his help on operating the microscope. We are also grateful of the help from Prof. Archie Howie (Department of Physics, Cambridge University) on discussions on the dielectric approach to EELS interpretation. Mr. Timothy Karcher performed the XPS tests on the commercial anatase powder. Financial support from US Department of Energy (DE-SC0004954) and the use of NION UltraSTEM at John M. Cowley Center for High Resolution Microscopy at Arizona State University are gratefully acknowledged. 


\section{References:}

[1] I. Tamm, About a possible type of electron binding on crystal surfaces., Z. Phys. 76 (1932) 849-850. doi:10.1007/BF01341581.

[2] V.E. Henrich, The surfaces of metal oxides, Rep. Prog. Phys. 48 (1985) 1481.

[3] R.O. Jones, Intrinsic Surface States in Semiconductors, Phys. Rev. Lett. 20 (1968) 992.

[4] J.D. Levine, P. Mark, Theory and Observation of Intrinsic Surface States on Ionic Crystals, Phys. Rev. 144 (1965) 751-763.

[5] G. Allan, C. Delerue, M. Lannoo, Nature of luminescent surface states of semiconductor nanocrystallites, Phys. Rev. Lett. 76 (1996) 2961.

[6] H.H. Kung, Transition Metal Oxides: Surface Chemistry and Catalysis, Elsevier, 1989.

[7] M. Henzler, The origin of surface states, Surf. Sci. 25 (1971) 650-680. doi:10.1016/00396028(71)90153-1.

[8] S.G. Davison, J.D. Levine, Surface States, Solid State Phys. - Adv. Res. Appl. 25 (1970) 1149. doi:10.1016/S0081-1947(08)60008-9.

[9] W. Mönch, Semiconductor Surfaces and Interfaces, Springer Science \& Business Media, 2013.

[10] M. Brause, S. Skordas, V. Kempter, Study of the electronic structure of TiO2(110) and $\mathrm{Cs} / \mathrm{TiO} 2(110)$ with metastable impact electron spectroscopy and ultraviolet photoemission spectroscopy (HeI), Surf. Sci. 445 (2000) 224-234. doi:10.1016/S0039-6028(99)01052-3.

[11] Z.L. Wang, J.M. Cowley, Reflection electron energy loss spectroscopy (REELS): A technique for the study of surfaces, Surf. Sci. 193 (1988) 501-512. doi:10.1016/00396028(88)90449-9. 
[12] V.E. Henrich, G. Dresselhaus, H.J. Zeiger, Energy-dependent electron-energy-loss spectroscopy: Application to the surface and bulk electronic structure of MgO, Phys. Rev. B. 22 (1980) 4764-4775. doi:10.1103/PhysRevB.22.4764.

[13] H. Ibach, D.L. Mills, Electron Energy Loss Spectroscopy and Surface Vibrations, Academic Press, 2013.

[14] Y. Harada, S. Masuda, H. Ozaki, Electron Spectroscopy Using Metastable Atoms as Probes for Solid Surfaces, Chem. Rev. 97 (1997) 1897-1952. doi:10.1021/cr940315v.

[15] J.A. Stroscio, R.M. Feenstra, A.P. Fein, Electronic structure of the Si (111) $2 \times 1$ surface by scanning-tunneling microscopy, Phys. Rev. Lett. 57 (1986) 2579.

[16] L. Kronik, Y. Shapira, Surface photovoltage spectroscopy of semiconductor structures: at the crossroads of physics, chemistry and electrical engineering, Surf. Interface Anal. 31 (2001) 954-965. doi:10.1002/sia.1132.

[17] O.L. Krivanek, T.C. Lovejoy, N. Dellby, T. Aoki, R.W. Carpenter, P. Rez, E. Soignard, J. Zhu, P.E. Batson, M.J. Lagos, R.F. Egerton, P.A. Crozier, Vibrational spectroscopy in the electron microscope, Nature. 514 (2014) 209-212. doi:10.1038/nature13870.

[18] D.A. Muller, L.F. Kourkoutis, M. Murfitt, J.H. Song, H.Y. Hwang, J. Silcox, N. Dellby, O.L. Krivanek, Atomic-Scale Chemical Imaging of Composition and Bonding by Aberration-Corrected Microscopy, Science. 319 (2008) 1073-1076. doi:10.1126/science. 1148820 .

[19] Q.M. Ramasse, C.R. Seabourne, D.-M. Kepaptsoglou, R. Zan, U. Bangert, A.J. Scott, Probing the Bonding and Electronic Structure of Single Atom Dopants in Graphene with Electron Energy Loss Spectroscopy, Nano Lett. 13 (2013) 4989-4995. doi:10.1021/nl304187e.

[20] R.F. Egerton, F. Wang, P.A. Crozier, Beam-Induced Damage to Thin Specimens in an Intense Electron Probe, Microsc. Microanal. 12 (2006) 65-71. doi:10.1017/S1431927606060065.

[21] R.F. Egerton, Control of radiation damage in the TEM, Ultramicroscopy. 127 (2013) 100108. doi:10.1016/j.ultramic.2012.07.006.

[22] R.F. Egerton, Mechanisms of radiation damage in beam-sensitive specimens, for TEM accelerating voltages between 10 and 300 kV, Microsc. Res. Tech. 75 (2012) 1550-1556. doi:10.1002/jemt.22099.

[23] P.E. Batson, New Surface Plasmon Resonance in Clusters of Small Aluminum Spheres, Ultramicroscopy. 9 (1982) 277-282. doi:10.1016/0304-3991(82)90212-1.

[24] A. Howie, R.H. Milne, Excitations at Interfaces and Small Particles, Ultramicroscopy. 18 (1985) 427-434.

[25]F.J. García de Abajo, Optical excitations in electron microscopy, Rev. Mod. Phys. 82 (2010) 209-275. doi:10.1103/RevModPhys.82.209.

[26] W. Langel, M. Parrinello, Hydrolysis at stepped MgO surfaces, Phys. Rev. Lett. 73 (1994) 504.

[27] A. Kudo, K. Domen, K. Maruya, T. Onishi, Photocatalytic Activities of TiO2 Loaded with NiO, Chem. Phys. Lett. 133 (1987) 517-519. doi:10.1016/0009-2614(87)80070-2.

[28] L. Zhang, Q. Liu, T. Aoki, P.A. Crozier, Structural Evolution during Photocorrosion of $\mathrm{Ni} / \mathrm{NiO}$ Core/Shell Cocatalyst on TiO2, J. Phys. Chem. C. 119 (2015) 7207-7214. doi:10.1021/jp512907g. 
[29] A.L. Linsebigler, G. Lu, J.T. Yates, Photocatalysis on TiO2 Surfaces: Principles, Mechanisms, and Selected Results, Chem. Rev. 95 (1995) 735-758. doi:10.1021/cr00035a013.

[30] A. Gibson, R. Haydock, J.P. LaFemina, Electronic structure and relative stability of the MgO (001) and (111) surfaces, J. Vac. Sci. Technol. A. 10 (1992) 2361-2366. doi:10.1116/1.577965.

[31] D.M. Roessler, W.C. Walker, Electronic Spectrum and Ultraviolet Optical Properties of Crystalline MgO, Phys. Rev. 159 (1967) 733-738. doi:10.1103/PhysRev.159.733.

[32] R. Egerton, Electron Energy-Loss Spectroscopy in the Electron Microscope, 3rd ed., Springer Science \& Business Media, 2011.

[33] P.E. Batson, Surface plasmon scattering on flat surfaces at grazing incidence, Ultramicroscopy. 11 (1983) 299-302.

[34] H. Raether, Solid state excitations by electrons, in: Springer Tracts Mod. Phys. Vol. 38, Springer-Verlag, Berlin/Heidelberg, 1965: pp. 84-157. http://www.springerlink.com/index/10.1007/BFb0045738 (accessed December 23, 2015).

[35] P. Moreau, N. Brun, C.A. Walsh, C. Colliex, A. Howie, Relativistic effects in electronenergy-loss-spectroscopy observations of the $\mathrm{S}$ i/S i O 2 interface plasmon peak, Phys. Rev. B. 56 (1997) 6774.

[36] J. Zhu, P.A. Crozier, P. Ercius, J.R. Anderson, Derivation of Optical Properties of Carbonaceous Aerosols by Monochromated Electron Energy-Loss Spectroscopy, Microsc. Microanal. 20 (2014) 748-759. doi:10.1017/S143192761400049X.

[37] R. Al-Gaashani, S. Radiman, Y. Al-Douri, N. Tabet, A.R. Daud, Investigation of the optical properties of $\mathrm{Mg}(\mathrm{OH}) 2$ and $\mathrm{MgO}$ nanostructures obtained by microwave-assisted methods, J. Alloys Compd. 521 (2012) 71-76. doi:10.1016/j.jallcom.2012.01.045.

[38] W.J. Bowman, K. March, C.A. Hernandez, P.A. Crozier, Measuring bandgap states in individual non-stoichiometric oxide nanoparticles using monochromated STEM EELS: The Praseodymium-ceria case, Ultramicroscopy. 167 (2016) 5-10. doi:10.1016/j.ultramic.2016.04.009.

[39] T. Bredow, A.R. Gerson, Effect of exchange and correlation on bulk properties of MgO, NiO, and CoO, Phys. Rev. B. 61 (2000) 5194.

[40] P.D.C. King, T.D. Veal, A. Schleife, J. Zúñiga-Pérez, B. Martel, P.H. Jefferson, F. Fuchs, V. Muñoz-Sanjosé, F. Bechstedt, C.F. McConville, Valence-band electronic structure of CdO, $\mathrm{ZnO}$, and $\mathrm{MgO}$ from x-ray photoemission spectroscopy and quasi-particle-corrected density-functional theory calculations, Phys. Rev. B. 79 (2009). doi:10.1103/PhysRevB.79.205205.

[41] A. Malashevich, E.I. Altman, S. Ismail-Beigi, Imaging the buried MgO/Ag interface: Formation mechanism of the STM contrast, Phys. Rev. B. 90 (2014). doi:10.1103/PhysRevB.90.165426.

[42] Y.-N. Xu, W.Y. Ching, Self-consistent band structures, charge distributions, and opticalabsorption spectra in $\mathrm{MgO}, \alpha-\mathrm{Al} 2 \mathrm{O} 3$, and MgAl2O4, Phys. Rev. B. 43 (1991) 4461-4472. doi:10.1103/PhysRevB.43.4461.

[43] V.V. Sobolev, Exciton spectra of MgO, Inorg. Mater. 40 (2004) 1169-1172.

[44] J. Kischkat, S. Peters, B. Gruska, M. Semtsiv, M. Chashnikova, M. Klinkmüller, O. Fedosenko, S. Machulik, A. Aleksandrova, G. Monastyrskyi, Y. Flores, W.T. Masselink, Mid-infrared optical properties of thin films of aluminum oxide, titanium dioxide, silicon 
dioxide, aluminum nitride, and silicon nitride, Appl. Opt. 51 (2012) 6789-6798. doi:10.1364/AO.51.006789.

[45] M.A. Henderson, W.S. Epling, C.H.F. Peden, C.L. Perkins, Insights into Photoexcited Electron Scavenging Processes on TiO2 Obtained from Studies of the Reaction of $\mathrm{O} 2$ with OH Groups Adsorbed at Electronic Defects on TiO2(110), J. Phys. Chem. B. 107 (2003) 534-545. doi:10.1021/jp0262113.

[46] E. Finazzi, C. Di Valentin, G. Pacchioni, A. Selloni, Excess electron states in reduced bulk anatase TiO2: comparison of standard GGA, GGA+U, and hybrid DFT calculations, J. Chem. Phys. 129 (2008) 154113. doi:10.1063/1.2996362.

[47] M. Calatayud, P. Mori-Sánchez, A. Beltrán, A. Martín Pendás, E. Francisco, J. Andrés, J.M. Recio, Quantum-mechanical analysis of the equation of state of anatase TiO 2, Phys. Rev. B. 64 (2001) 184113. doi:10.1103/PhysRevB.64.184113.

[48] X. Liu, G. Chen, J.G. Erwin, N.K. Adam, C. Su, Release of phosphorous impurity from $\mathrm{TiO} 2$ anatase and rutile nanoparticles in aquatic environments and its implications, Water Res. 47 (2013) 6149-6156. doi:10.1016/j.watres.2013.07.034.

[49] P.J. Hardman, R. Casanova, K. Prabhakaran, C.A. Muryn, P.L. Wincott, G. Thornton, Electronic structure effects of potassium adsorption on TiO2(100), Surf. Sci. 269 (1992) 677-681. doi:10.1016/0039-6028(92)91331-5.

[50] K.K. Adepalli, M. Kelsch, R. Merkle, J. Maier, Enhanced ionic conductivity in polycrystalline TiO2 by "one-dimensional doping," Phys. Chem. Chem. Phys. 16 (2014) 4942-4951. doi:10.1039/C3CP55054K.

[51] U. Diebold, The surface science of titanium dioxide, Surf. Sci. Rep. 48 (2003) 53-229. doi:10.1016/S0167-5729(02)00100-0.

[52] A. Fujishima, X. Zhang, D.A. Tryk, TiO2 photocatalysis and related surface phenomena, Surf. Sci. Rep. 63 (2008) 515-582. doi:10.1016/j.surfrep.2008.10.001.

[53] M.A. Henderson, J.M. White, H. Uetsuka, H. Onishi, Photochemical Charge Transfer and Trapping at the Interface between an Organic Adlayer and an Oxide Semiconductor, J. Am. Chem. Soc. 125 (2003) 14974-14975. doi:10.1021/ja037764+.

[54] M. Landmann, E. Rauls, W.G. Schmidt, The electronic structure and optical response of rutile, anatase and brookite $\mathrm{TiO}_{2}$, J. Phys. Condens. Matter. 24 (2012) 195503. doi:10.1088/0953-8984/24/19/195503.

[55] Z. Zhao, Z. Li, Z. Zou, Surface properties and electronic structure of low-index stoichiometric anatase $\mathrm{TiO}_{2}$ surfaces, J. Phys. Condens. Matter. 22 (2010) 175008. doi:10.1088/0953-8984/22/17/175008.

[56] A. Gloter, M.-W. Chu, M. Kociak, C.H. Chen, C. Colliex, Probing non-dipole allowed excitations in highly correlated materials with nanoscale resolution, Ultramicroscopy. 109 (2009) 1333-1337. doi:10.1016/j.ultramic.2009.06.005.

[57] J.M. Cowley, Surface energies and surface structure of small crystals studied by use of a stem instrument, Surf. Sci. 114 (1982) 587-606. doi:10.1016/0039-6028(82)90707-5. 

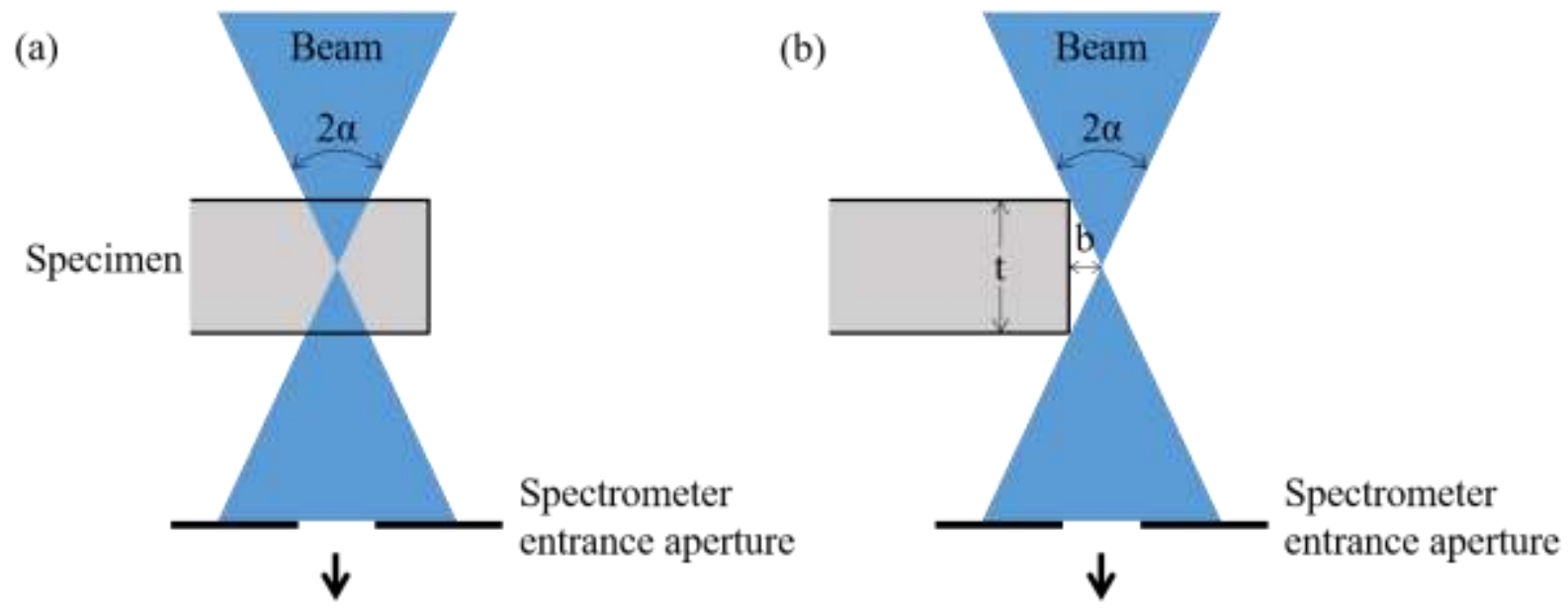

Figure 1: Schematic diagram showing the geometry of (a) transmission and (b) aloof convergent beam configuration. 

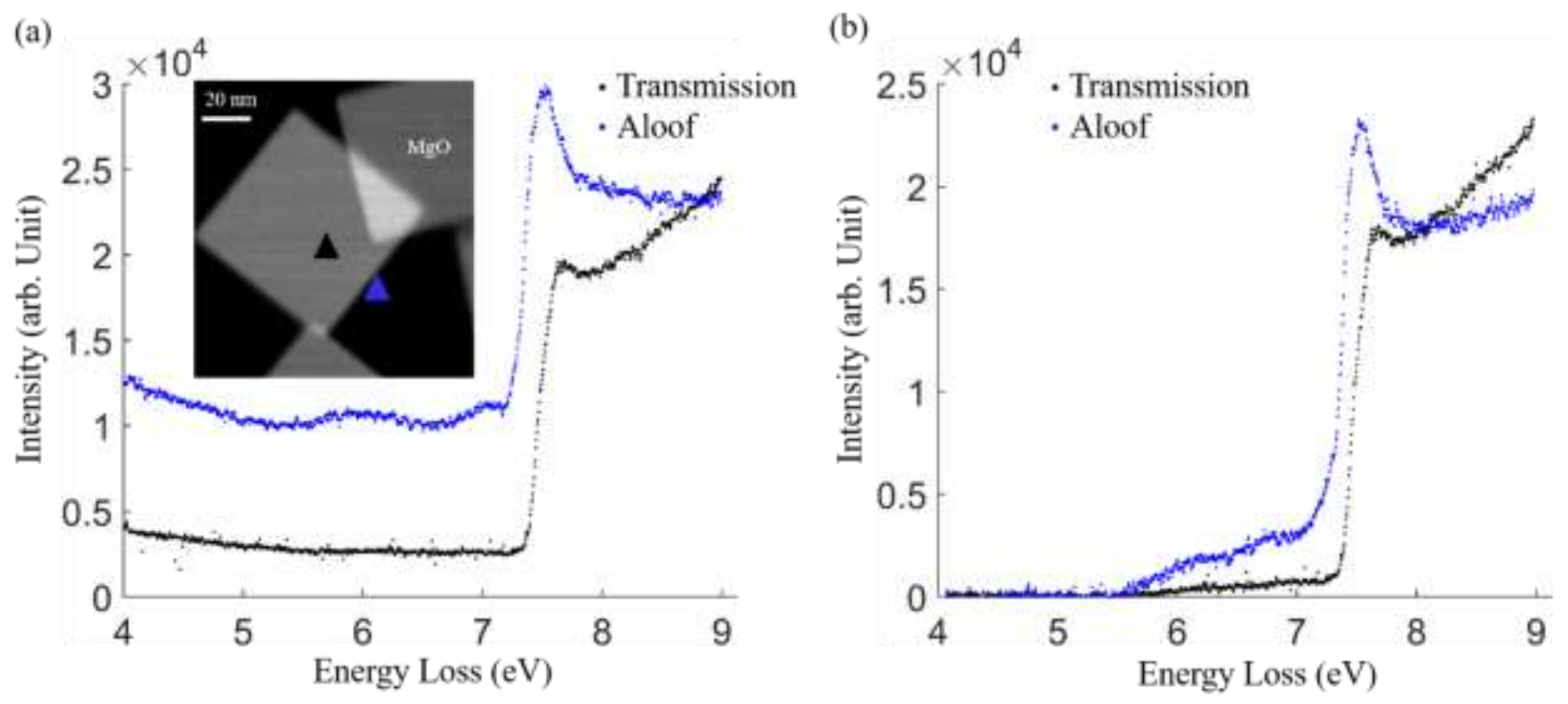

Figure 2: (a) HAADF image showing $\mathrm{MgO}$ nanocubes and beam positions for transmission (black triangle) and aloof (blue triangle) configurations. Raw spectra from the two positions are plotted. (b) Background removed transmission and aloof spectra. 
(a)

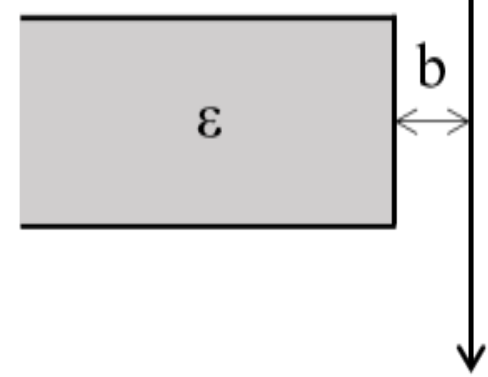

(b)

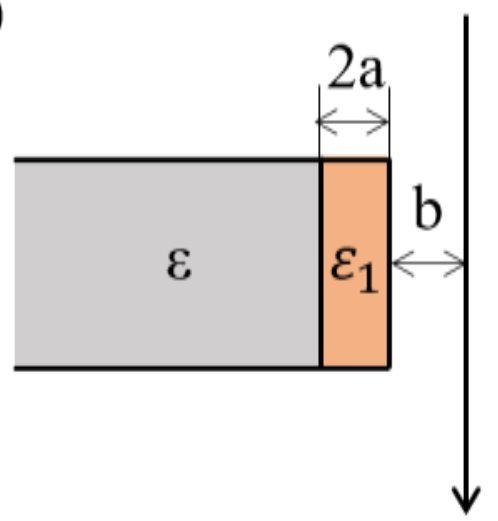

Figure 3: Schematic drawing of (a) a dielectric bulk terminated structure with a parallel beam outside the sample, at a distance b. (b) a dielectric model of substrate $(\varepsilon)$ and surface $\left(\varepsilon_{1}\right)$ structure with a parallel beam at a distance b. The surface layer thickness is $2 \mathrm{a}$.

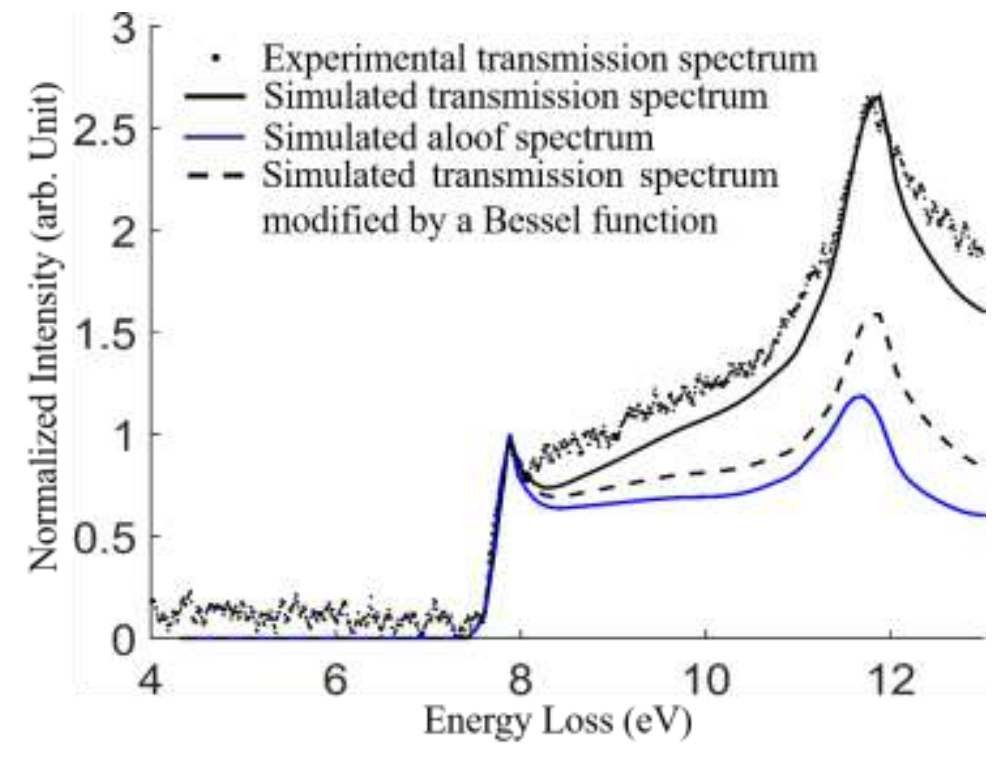

Figure 4: Experimental (dotted) and simulated (solid black) $\mathrm{MgO}$ bandgap onset in transmission mode. Simulated aloof mode spectrum (blue) using dielectric model, and simulated transmission multiplied by a Bessel function (dashed black) are also shown. All are normalized to set the intensity at $7.9 \mathrm{eV}$ to be 1 unit. 

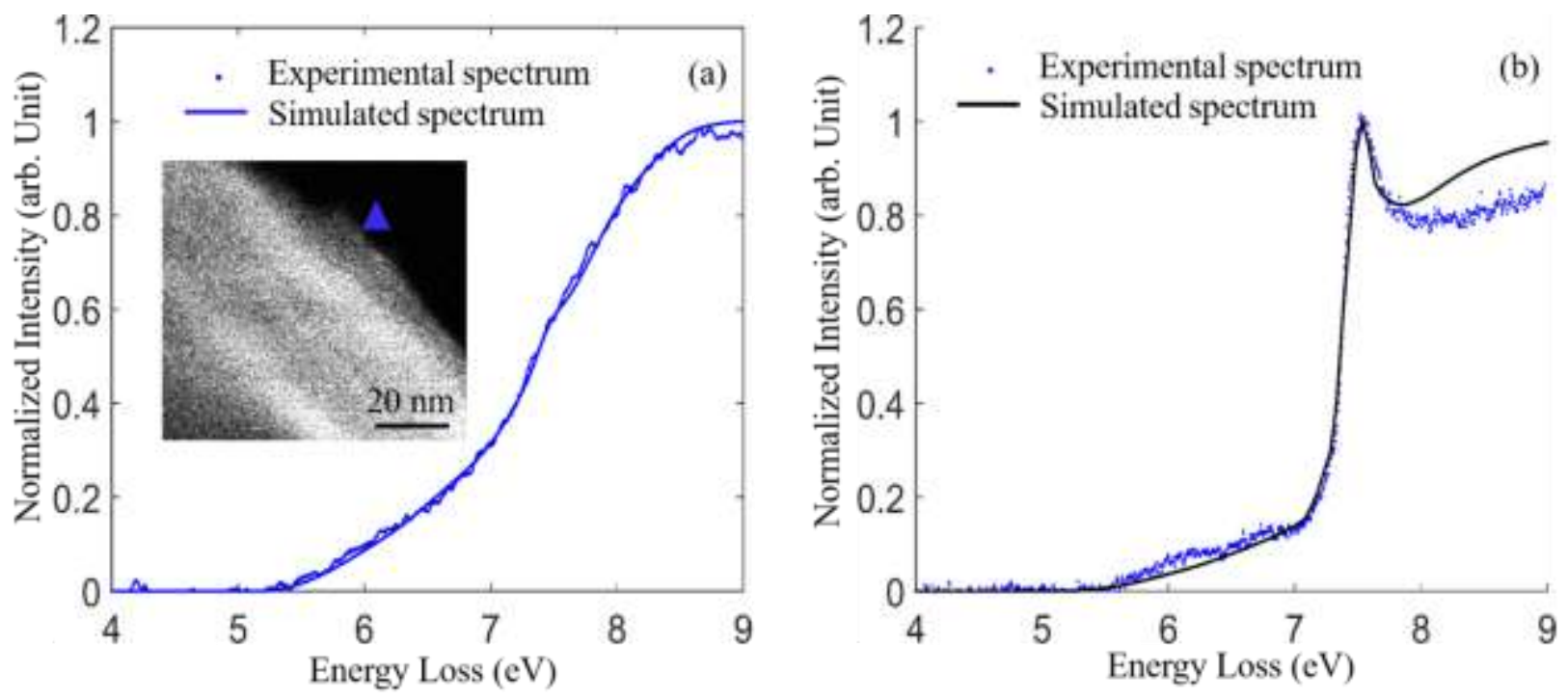

Figure 5: (a) Experimental and simulated bandgap onsets of $\mathrm{Mg}(\mathrm{OH})_{2}$ when the beam is $3 \mathrm{~nm}$ away from the sample surface. (b) Simulated aloof energy-loss spectrum for a $1.7 \mathrm{~nm}$ overlayer of $\mathrm{Mg}(\mathrm{OH})_{2}$ on $\mathrm{MgO}$ compared with the experimental aloof spectrum. 

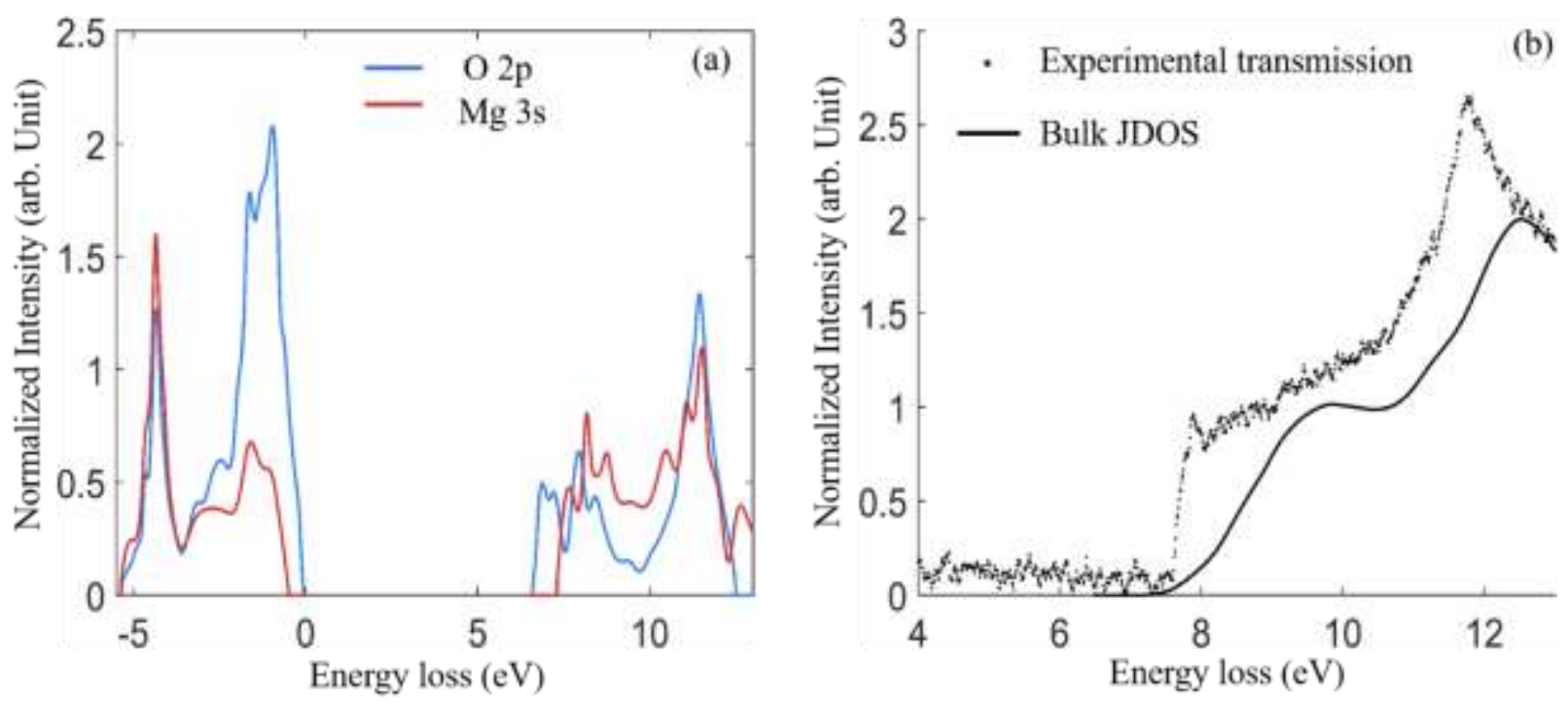

Figure 6: (a) Modified PDOS of MgO, with top of valence band set to $0 \mathrm{eV}$. (b) Experimental bulk spectrum and calculated bulk JDOS. 

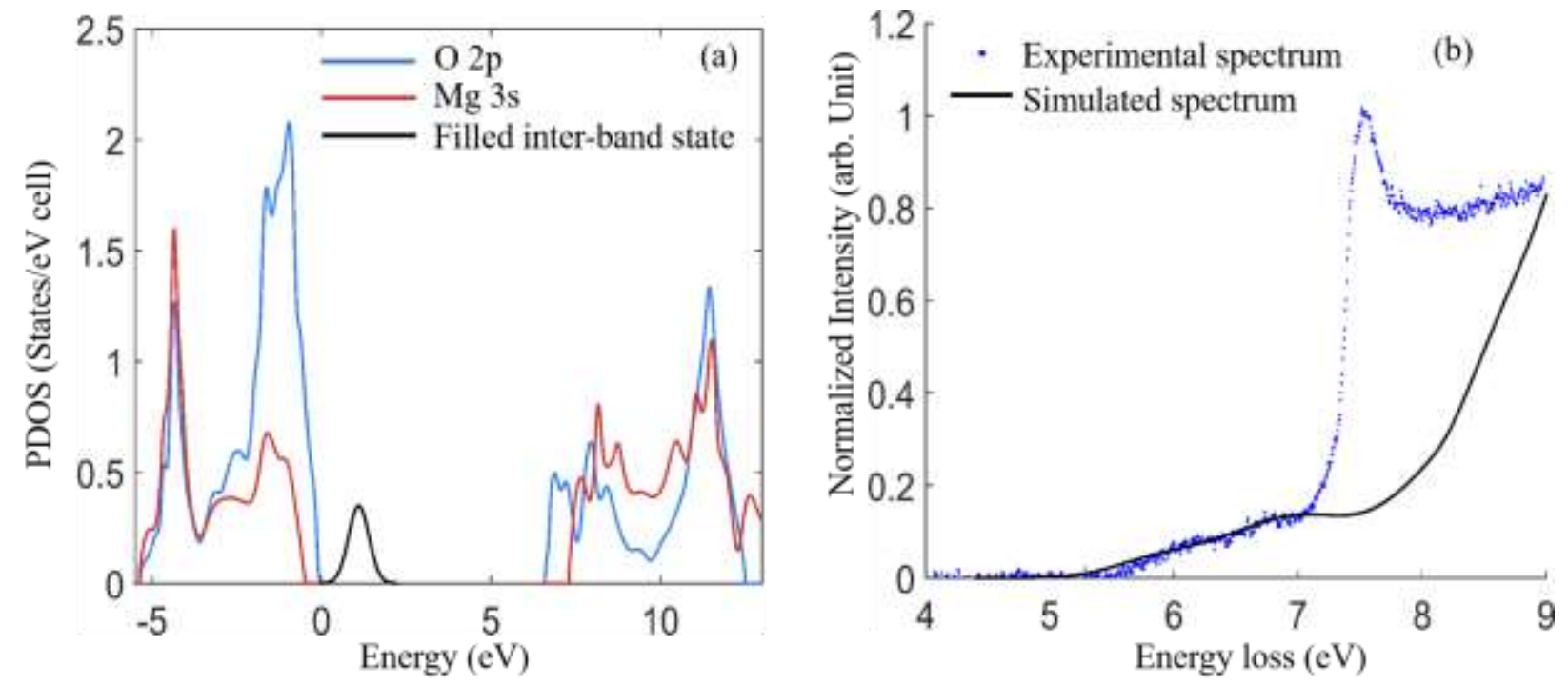

Figure 7: (a) PDOS of $\mathrm{MgO}$ with a Gaussian shaped filled state centered at $1.1 \mathrm{eV}$ above the valence band with FWHM of $0.7 \mathrm{eV}$. (b) Black curve is the simulated spectrum from the DOS in a), compared with the experimental aloof spectrum (dotted blue). 


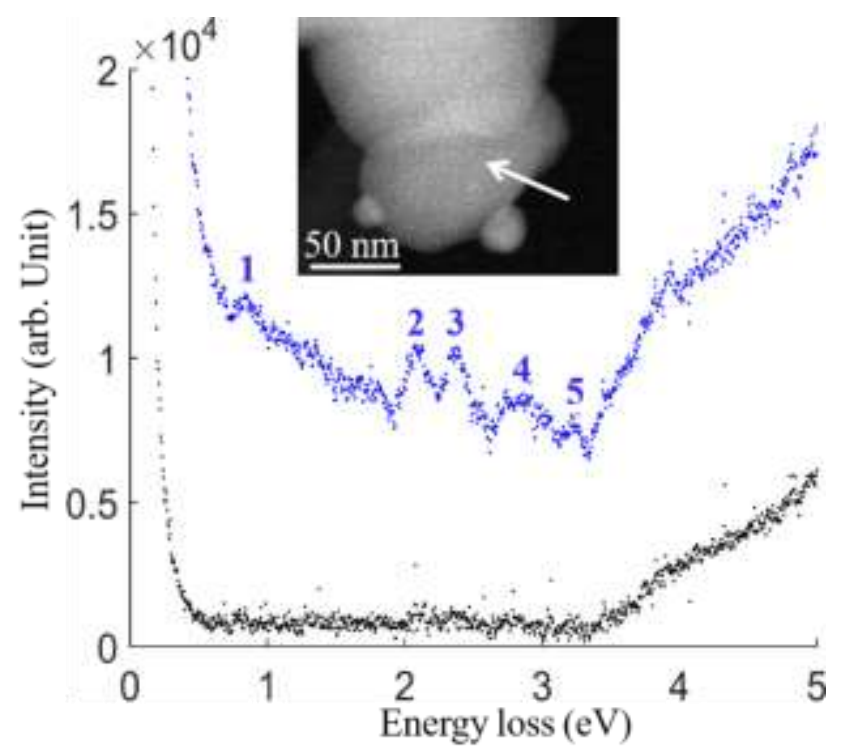

Figure 8: Transmission (black) and aloof (blue) spectra extracted from a line scan going from the vacuum into anatase particle. Insert shows a HAADF image of anatase particle supporting two Ni metal particles and the line scan trajectory. 

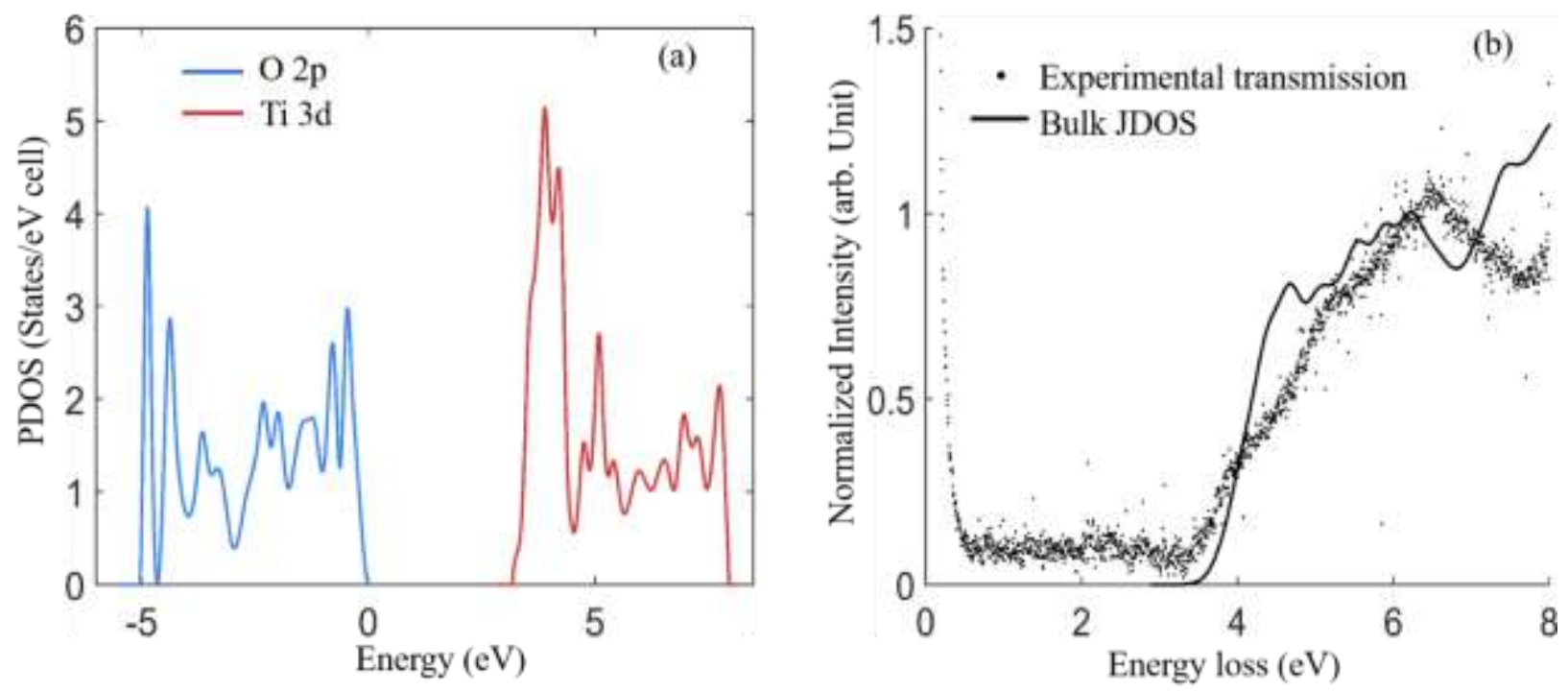

Figure 9: (a) Modified PDOS of anatase. (b) Bulk JDOS of a) compared to experimental transmission spectrum of anatase. 

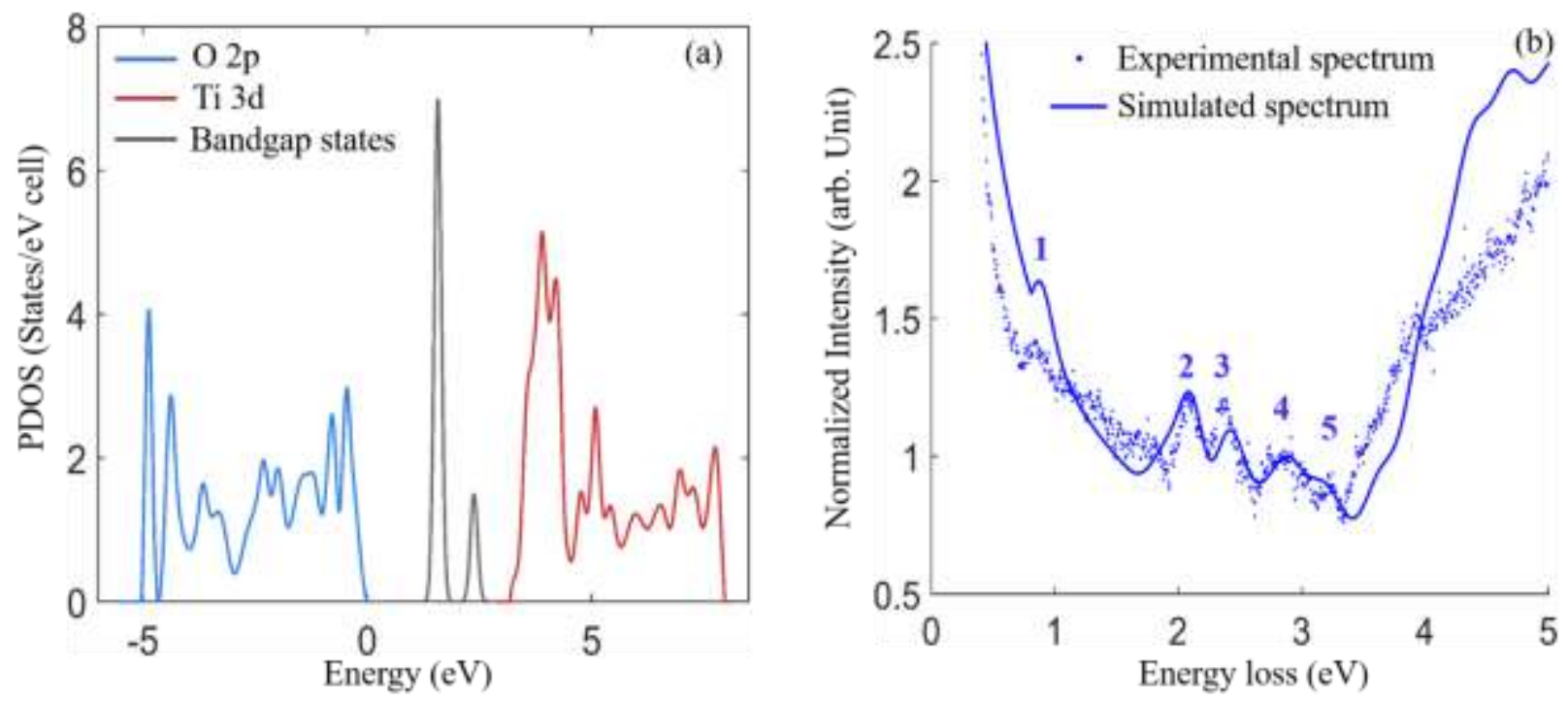

Figure 10: (a) PDOS of anatase with Gaussian shaped states center at 1.6 and $2.4 \mathrm{eV}$ above the valence band with FWHM of $0.19 \mathrm{eV}$. (b) Simulated spectrum from the DOS in a) overlaid with the experimental spectrum. A simple powerlaw background has been added to the simulation as a guide to the eye for comparing simulation with experiment. 

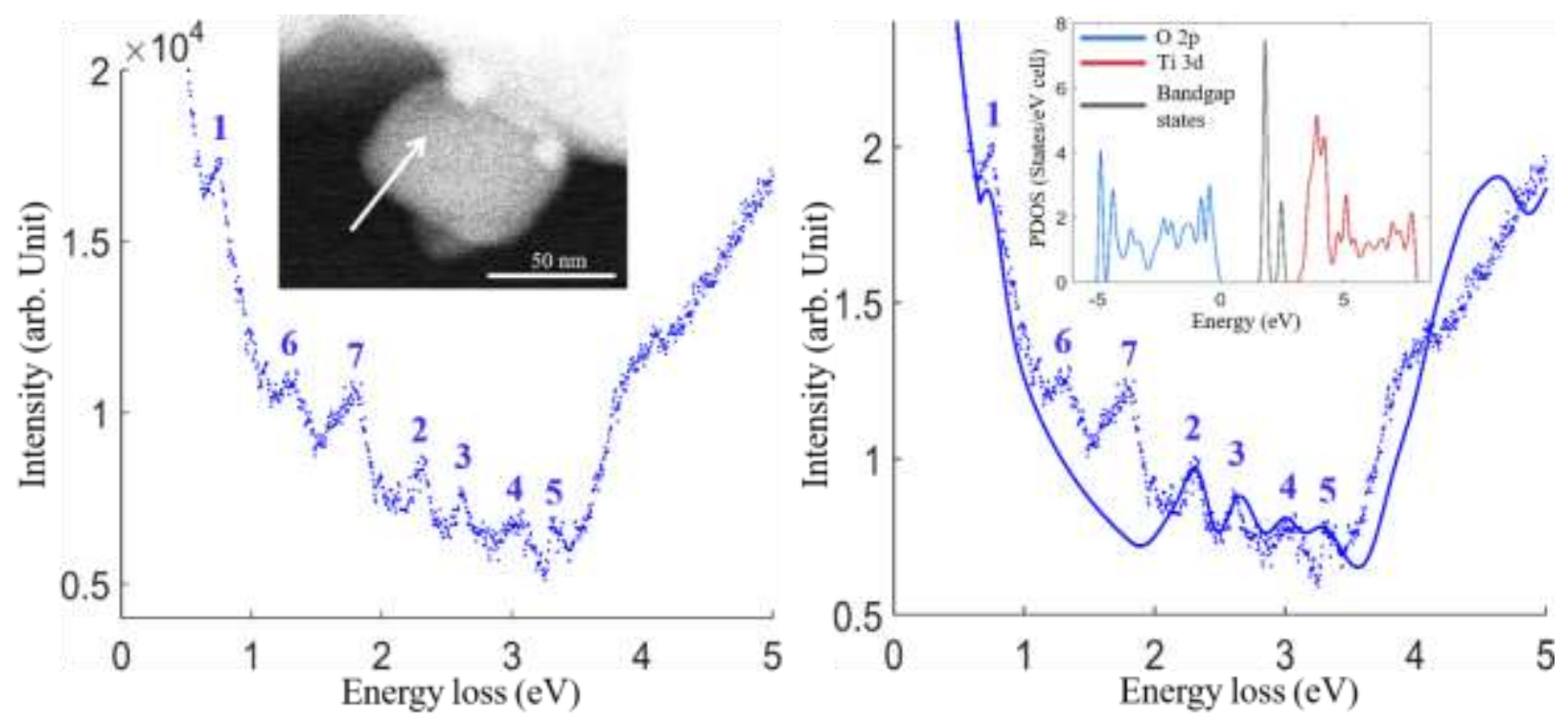

Figure 11: (a) Extracted aloof spectrum from a line scan going from the vacuum into an anatase particle. Insert shows the HAADF image of this anatase particle and the line scan trajectory. (b) Simulated spectrum overlaid with the experimental spectrum. Insert is the PDOS used for the simulation, with Gaussian shaped bandgap states center at 1.8 and $2.5 \mathrm{eV}$ above the valence band with FWHM of $0.19 \mathrm{eV}$. 